\title{
The experience of the illness and of the treatment for the person with systemic arterial hypertension: an ethnographic study ${ }^{1}$
}

\author{
Silvana Maria Coelho Leite Fava ${ }^{2}$ \\ Márcia Maria Fontão Zago ${ }^{3}$ \\ Maria Suely Nogueira ${ }^{3}$ \\ Eliza Maria Rezende Dázio ${ }^{4}$
}

\begin{abstract}
Objective: to interpret the meanings of the experience of the condition and of the treatment among people with arterial hypertension. Method: the authors adopted the frames of reference of interpretive and medical anthropology and of the ethnographic method. 22 people with arterial hypertension, and 10 Family Health Strategy health workers, all from Minas Gerais, participated. The authors used interviews, participant observation, focus groups, field diaries and analysis of medical records. Ethical precepts were respected. Results: two nuclei of meaning emerged: "The condition as an expression of way of living", and "The perspective of the cure of the condition". Nervous problems represent the nosological and symptomatic categories, caused by the urban way of living. The participants are supported by the belief of the curing of the problem. The family, spirituality and religion constitute social support networks. The therapeutic routes interpenetrate for the cure of the problem. The 'folk' health subsystem constitutes an important route because it provides better well-being and remission of the symptoms. Conclusion: the gaps evidenced between the points of view of the health professionals and the interviewees allow one to rethink the praxis so as to provide comprehensive, contextualized and humanized care, which encourages the people's potential for living, for empowerment, and for self-care.
\end{abstract}

Descriptors: Hypertension; Culture; Nursing; Anthropology, Medical; Patient Compliance.

\footnotetext{
${ }^{1}$ Paper extracted from doctoral dissertation "Os significados da experiência da doença e do tratamento para a pessoa com hipertensão arterial e o contexto do sistema de cuidado à saúde: um estudo etnográfico" presented to Escola de Enfermagem de Ribeirão Preto, Universidade de São Paulo, WHO Collaborating Centre for Nursing Research Development, Ribeirão Preto, SP, Brazil. Supported by Conselho Nacional de Desenvolvimento Científico e Tecnológico (CNPq), process \# 143026/2009-7.

${ }^{2} \mathrm{PhD}$, Associate Professor, Escola de Enfermagem, Universidade Federal de Alfenas, Alfenas, MG, Brazil.

${ }^{3}$ PhD, Associate Professor, Escola de Enfermagem de Ribeirão Preto, Universidade de São Paulo, WHO Collaborating Centre for Nursing Research Development, Ribeirão Preto, SP, Brazil.

${ }^{4} \mathrm{PhD}$, Adjunct Professor, Escola de Enfermagem, Universidade Federal de Alfenas, Alfenas, MG, Brazil.
}

Corresponding Author:

Silvana Maria Coelho Leite Fava

Universidade Federal de Alfenas. Escola de Enfermagem

Rua Gabriel Monteiro da Silva, 700

Centro

CEP: 37130-000, Alfenas, MG, Brasil

E-mail: silvanalf2005@yahoo.com.br 


\section{Introduction}

At the present time, nursing is facing great challenges, including dealing with the complexity of the human being with chronic conditions such as Systemic Arterial Hypertension (SAH). SAH is a multifactorial clinical condition, characterized by high and sustained levels of arterial pressure (AP). Its clinical criteria, in individuals over 18 years of age, are tensional levels equal to or greater than $140 \mathrm{mmHg} \times 90 \mathrm{mmHg}^{(1)}$. $\mathrm{SAH}$ has specific characteristics which influence the perception and the co-existence of the person with the illness. The chance nature of the discovery of the condition, the atemporality of the phases of remission and exacerbations ${ }^{(2)}$, the long period of latency and the long period of time with no symptoms ${ }^{(3)}$, the label of the condition which has a negative effect on well-being(4), accompanied by manifestations and loss of social wellbeing ${ }^{(5)}$, and the medicalization(6) bring significant repercussions on how life is seen. In this way, the condition changes the relationship the person has with themselves and with the world, which influences the reconstruction of their identity. SAH causes significant transformations in the people's lives, in the psychological, family, social or economic spheres, due to the possibility of a longterm health problem. These transformations back up the idea that questions relevant to the condition cannot be analyzed in isolation from the other dimensions of social life, mediated and permeated by culture, which confers meaning on these experiences ${ }^{(7)}$. In the care for the person with $\mathrm{SAH}$, the nurse needs to participate in the processes of construction of knowledge so as to assimilate and use innovations - whether technological or humanistic - in a balanced way, such that she may offer the person conditions to carry out self-care in the way that is most satisfactory to their life context. Throughout their professional activities, as lecturers and an extension project coordinator, based on actions grounded in the principle of the indissociability of teaching, research and extension, with views to education and health care for people with $\mathrm{SAH}$, and on studies resulting from these actions ${ }^{(8-10)}$, the authors have identified that problems related to adherence to treatment are not restricted only to the physical sphere and to pharmacological therapy - also implicit are life experience and the subjectivity in the process of becoming ill and caring for oneself. The authors noticed that the ways of thinking and acting of people with $\mathrm{SAH}$, which may be translated by the experience of the illness, are understood and valued little by the health professionals. We observed that these studies partially respond to our concerns, as we continue to experience situations which create expectations and distress which translate into the following questions: How do they interpret the condition? How do they experience falling ill? How is the healthcare system shaped? In order to respond to these concerns, we sought to combine other frames of reference besides the biological one, as it is recognized that the experience of the condition and the actions necessary for the adherence to treatment and care in the long term are profoundly interwoven with the culture - that is, with how people live, with their habits, routines, and the rituals in their lives.

In interpretive anthropology(11) and medical anthropology we found frames of reference which enabled us to find answers, as these have as objectives to observe, describe, analyze and interpret cultural systems based on the viewpoints of their members. Anthropology is considered a science of difference and diversity, which allows one to develop a critical perspective in the face of our more fundamental 'truths', favoring the construction of a new approach. It is concerned with studying the characteristics of people in society, with a focus on the culture, with its systems of symbols, ideas and meanings, which allows one to understand human behavior in a broader way, taking into consideration life's various dimensions. Anthropology brings important contributions to the functioning of the nurse because it makes it possible to analyze how the person lives in their daily life, their interpretations regarding the health-illness process, the ways of living and the treatment choices. As regards medical anthropology, one of its main exponents(12) describes the "professional" and "cultural" constructions of condition in his Explanatory Model (EM). One finds the distinction between disease "condition-process" as the abnormalities of the biological and/or psychological processes, and the illness - the "experienced condition", which refers to the psycho-social experience of the condition, which is centered in the subjectivity, which includes the condition's cultural, social and personal elements. We learned that the experience of the condition is molded by the socio-cultural context which determines cultural and symbolical forms of expression for interpreting the condition, the way in which one feels and expresses one's symptoms, uses curative resources and stops these from changing one's way of living. The search for treatment for SAH depends on the meanings constructed throughout life, which often come into confrontation with the actions prescribed by the health 
professionals. We recognize that living with $\mathrm{SAH}$ is a complex experience, given the need for medicalization of life, given the abdications from the everyday, and the need for interactions with the health-care subsystems throughout life, to treat the condition or for the care which is intrinsic to their living conditions. In spite of the growing interest from research for understanding the cultural construction of the health-illness process, there are few current works related to SAH in this perspective ${ }^{(13-16)}$. The scarcity of works anchored in this frame of reference and the search for answers to our concerns, which involve how people with SAH think, feel and do in order to co-exist with the condition and the search for treatment explain the development of this work, which aims to interpret the meanings of the experience of the condition and of the treatment of the people with $\mathrm{SAH}$ registered in a Family Health Strategy Center in a city in the state of Minas Gerais.

\section{Methods}

This is interpretive research, based in the frame of reference of interpretive anthropology ${ }^{(11)}$, medical anthropology(12) and the ethnographic method, ethnography being understood here as the search for a dense description(11). Ethnography, having as its principles the prolonged and dialogic relationship with the interviewees, being open to knowledge of 'common knowledge', in an in-depth view of the context under investigation, allowed us to decipher the codes which give meaning to the way of thinking and acting of the interviewees living with $\mathrm{SAH}$, in a general and contextualized view of the reality; and which further allowed the researchers and the interviewees, the exchange, the involvement, and the transformation of both. 22 interviewees with SAH participated in the study, both male and female, aged 18 or over, able to respond to the questions, registered on the Ministry of Health's HIPERDIA computer system, from an area associated to a Family Health Strategy Center (UESF), as did 10 health workers from the same center in a city in Minas Gerais. The data was collected in the period April 2010 - November 2011, through: immersion in the field; the collection of statements; participant observation; field diaries; and analysis of medical records. The collections of series of statements were made by the first author through previously-arranged home visits. The accounts were recorded on a Powerpack digital voice recorder and were transcribed immediately afterwards, which allowed them to be complemented with observations and notes from the field diary. After the production of the texts, these were read and, later, compared with the recordings. For collecting statements, as the basic script we used the $\mathrm{EM}^{(12)}$ which seeks to explain the illness based on five basic questions: 1) etiology; 2) time since the symptoms appeared and how they did; 3) physiopathology; 4) the course of the illness; 5) treatment. Analysis was carried out concomitantly with the collection, which allowed us to identify contrasts and similarities, creating reflections on possibilities to refine questions, deepen them, and to return to the field for better interpretation of the meanings of the experience of the condition in the perspective of the person with $\mathrm{SAH}$. We did the following stages(17): organization of the data, based on the transcription of the accounts and the field diary; codification of the data, considering each line and phrase of the empirical material; and grouping of similar and contrasting codes to create the units of meaning, or empirical categories. These categories were named, using the interviewees' terminology. We selected the words or phrases which constituted the units of meaning, regrouped them according to the similarities, and codified them with significant expressions which translate the meanings of the interviewees' experience. In this way we constructed the nuclei of meaning, or analytical categories. As the analytical categories were created, we compared them against the empirical categories in the search for inter-relations and interconnections. This phase is complex, as, at the same time as it depends on the lens of the researcher in the interpretation process, it requires the exercising of otherness in an attempt to grasp the meanings attributed by the people with $\mathrm{SAH}$ for the interpretation of the meanings of the experience in the process of becoming ill. The reflexivity to interpret the meanings in the search for the significance was guided by the theoreticalmethodological frame of reference, by the ethnographic meeting, and by means of the understanding of the interviewees' historical context, in which the meanings are produced, seeking to decode them so as to grasp the implicit meanings in the experience with falling ill. In analyzing the data, we find divergences and similarities and, based on the estrangement, it was possible to understand the other and re-think our way of thinking and acting. The study was approved by the Research Ethics Committee of the Federal University of Alfenas, under process No 018/2010. To participate in the study, the people were advised about the research's objectives, anonymity, and the signing of the Terms of Free and Informed Consent. 


\section{Results and Discussion}

Of the 22 interviewees with $\mathrm{SAH}$, thirteen were women and nine, men, aged between 34 and 84 years old. They were predominantly Caucasian, married and with children, and most were Roman Catholics, followed by Pentecostalists. Level of schooling was low, as was the monthly income: for the majority it was one minimum salary. Fifteen were retired, of whom four had retired for reasons of disability. The level of relatedness between the inhabitants is high, and there is similarity in their life trajectories; they were born, lived, married and raised their children in this community, working as builders, laborers, house-wives and maids. The health workers were five community health workers, one nurse, one doctor, one nursing auxiliary, one pharmacy assistant and one secretary. The data was organized and coded in seven units of meaning: "Discovery of the condition"; "The process of falling ill"; "Co-existence with the condition"; "Coping strategies"; "Beliefs"; "Therapeutic route"; and "Adherence to the treatment". After being interpreted, these resulted in the nuclei of meaning: "The condition as expression of the way of living" and "The perspective of cure of the condition". "The condition as expression of the way of living". We observed that the meanings attributed to the experience of the illness were marked by the chance nature of the discovery of the condition, which explained the anger and mistrust in the light of the diagnosis of the condition. [...] I feel a victim of chance. I went to the outpatient department for a consultation about something else and during the triage they checked my pressure and asked me if I had high pressure, and I said not, because I'd never felt anything, and that was when they labelled me as hypertensive, my pressure was 17/11, I didn't feel anything. But I was going through a lot of emotional problems (Wellington, 54 years old). Others, on the other hand, in the face of the perception that something is not right, break the silence of the assymptomatic illness ${ }^{(3)}$ because of the presence of unspecified manifestations which constitute cultural and symbolical forms of the experience they are living through. We notice that some dimension of the corporeality was compromised as a necessity for them to re-affirm the condition(18), as we observe in the account: [...] I felt bad, weak, dizzy, general ill feelings, head-ache, I didn't know it was my blood pressure, I thought it was anemia [...] so I went to the doctor at the clinic and they said it (pressure) was high. (Maria Aparecida, 55 years old). Nervous problems constituted the nosological category for the social group researched, disparate from the findings in the literature, and culturally molded by how they perceive, experience and deal with it [...] it is a nervous problem (pause) it's from getting really angry. On my street, it's too rowdy, too many fights, it doesn't let you relax, it's day and night (João, 79 years old). In relating the meaning of the word(19) "problem" to the conceptions which they have of SAH, we can identify similarities, and such definitions correspond to the meanings which the interviewees attribute to the condition, something difficult to explain, to deal with and to treat, which are expressed in feelings of doubt, uncertainty, anxiety and even fear of the condition. In relation to the causes, while for biomedicine these are related to malfunction of the blood vessels, the heart and the blood(1), for the interviewees they are related to nerves. The urban way of living constitutes the principal determinant for nerves. We learnt that the nervous problem incorporates a series of afflictions in the symptomatology and in the etiology and which are reflected in the experiences with the problems of everyday life, resulting from: financial difficulties; worries about the family; social isolation; problems from illnesses; alcoholism; marital and family conflicts; the loss of loved ones; dissatisfaction with the formal health system; the process of acculturation; and problems in the street. Our interpretations are in line with the conceptions of the author ${ }^{(12)}$, in considering the condition as a somatic way of experience and social suffering. It translates how a person experiences their sorrows, and their social suffering, which includes any type of problem. Nerves, at the personal level, represents a set of manifestations resulting from psycho-biological phenomena - and such manifestations suffer a cultural influence, as it is in this field that the stressing agents are perceived, labelled, explained and evaluated(12). Regarding the complications, for the people with $\mathrm{SAH}$ these are infarction, strokes and sudden death, while for biomedicine, these are cardiac insufficiency, renal insufficiency and the cerebrovascular accident ${ }^{(1)}$. The way that the interviewees - influenced by their sociocultural context - co-exist with the process of becoming ill allows them to express feelings and reactions whose interpretations are related to resiliency, resignation, low self-esteem and anger. [...] I think we are like a plant, we throw earth on it $[. .$.$] , the more we water it, the longer it lives,$ and that's how it is with us [...] you need medicines to preserve your life, when you care more, you live longer, and that's how life is too, you can't lead it any other way (Manoel, 84 years old). We recognize that the scars caused by problems in life which resulted in the nervous problem provided, for some, a new way of seeing the world and of re-signifying the care for health. We perceived that the beliefs 
influenced the interpretation which the interviewees had on the condition, guided their search for a cure, in the different health subsystems, and led them to weave the networks as support for facing falling ill. The beliefs also explained the living habits, the sexual impotence caused by the hypotensives, health and illness, the mistrust in relation to the composition of the drugs, the conception that medicine from the pharmacy does not cure, and that what is free of charge doesn't work.

"The perspective of cure of the condition". One aspect which was specific to the interviewees was the meanings attributed to the objectives of the treatment for SAH. Their perception of the problem is that it results from nerves, is symptomatic, and is curable; the treatment, therefore, according to their criteria, will be for a specified length of time. Among the interviewees, there are those who, after stopping with the treatment, maintain or occasionally present pressure levels within normal parameters, which led them to believe in the cure of the problem, as in the following account: [...] so the doctor told him (the spouse) to take the medicine, very well, he took it for a few days, he went there, (the ESF Center) measured his pressure and it was good, so he stopped with the medicines, because the pressure had got better (smiles). Mine hasn't, yet, I don't know why, but it will! (Gerusa, 60 years old). We learnt that the knowledges which circulate among them support behaviors and feelings in the process of living and of becoming ill. We evidenced that the experience of cure is shared and influences the abandoning of treatment. We perceived that the people with SAH seek a cure for the illness (healing) rather than a cure for the pathology (curing)(20). In spite of the author's emphasizing that the objective of curing is the remission of symptoms, in our study, the cure termed healing is also obtained by the remission of symptoms, as the problem is presented in a symptomatic form. Based on the data found, we recognize the importance of valuing the knowledge from 'common knowledge' on the experience of falling ill, with a view to facilitating the process of interaction and the negotiation of the therapeutic plan. In comparing the EMs between the health professionals and the people with $\mathrm{SAH}$, we observe that for the former, the condition is a functional interpretation, while for the latter, it is a symbolic interpretation, a harm which is more existential than functional. There are also divergences in relation to the treatment's goals and the evaluation. The divergence between the different points of view can negatively influence compliance with the treatment, satisfaction, and use of the health institutions ${ }^{(20)}$ and also leads them to a low perception and to greater vulnerability(21). In understanding that the nervous problem constitutes a harm whose nature is more existential than functional, we understand that different needs were compromised, (which led them to seek treatment in different health subsystems, as much to care for the mind as for the body. The family health subsystem constituted the primary source for interpretation of the manifestations of falling ill, treatment and counselling and both this and the 'folk subsystem' constituted the main supports for coping with the nervous problem. We perceived that the practices which are related to folk remedies are desired and used by the interviewees, because these are the resource which is the closest to, and is most commensurate with, the local culture, because of the sensation of well-being produced by the medicine or blessings ${ }^{1}$, because of the encounter with the lay healer, because of the mistrust in relation to the composition of the drugs, and because of the difficulty in accessing the formal health system, as we can see from the following statement: [...] it's difficult you know, we have to take care of ourselves, because there ain't no doctor for us to see! (Helena, 55 years old). Regarding adherence to the treatment, we noticed that the main difficulties were related to being tired of having to take so much medication, the mistrust in relation to the composition of the drug, the failure to value allopathy, the toxicity caused by the medication, the difficulties accessing the health system and the weak link had with it, the economic conditions, the belief that anything given for free can't be any good, the undesirable effects of the hypotensive medications which interfere with daily life, socialization and masculinity, forgetting to take the medications, hypotensives and alcoholic beverages: a fatal mixture, illiteracy, disbelief regarding the chronic nature of $\mathrm{SAH}$, and the postponement of starting treatment, because one has the rest of one's life to be treated, so one may as well start tomorrow. Even considering the difficulties described for the pharmacological treatment, many people still do it, even if this is in their own way, because it does not require changes in living habits, because it provides rapid relief from the manifestations, and, in observing that the problem results from nerves, attributed to the urban way of living, contributes to attributing the emotional state to the others, and exempting the sufferer from the responsibility for the

\footnotetext{
1 'Blessing' in this case is a simple ceremony performed by a local person regarded as being a 'healer'. Translator's note.
} 
control of the arterial pressure. On the other hand, regarding non-pharmacological treatment, we noticed that pork, pork fat and salt are powerful symbols of local and regional identity, among others, deeply rooted among the interviewees, for which reason it is very difficult to change the living habits. We perceived that falling ill causes the person to seek different coping strategies so as to overcome the adversities caused by the condition and by the treatment. This search is owed to the fact that the condition constitutes a harm to the totality of existence, as it is not the arterial pressure which is an annoyance - rather, it is the totality of the person which suffers( ${ }^{(22)}$. We observed that for the informants, religion, spirituality and family constituted the principal sources of support. Religion and spirituality had a positive influence on the nervous problem, by providing the ill person with better physical and subjective well-being; as well as contributing to behavioral changes and health care, they widened the social network and allowed a re-signification of life. We noticed that the interviewees' experience with the formal health system revealed feelings of outrage, dissatisfaction and anger, resulting from the difficulties related to the organizational and functional structure. The lack of medical attendance was one of the main causes of dissatisfaction with the health service. As a result of this, the people are motivated to 'do it their way' - that is, to seek support for treatment in the private/ professional, family or folk health subsystems. [...] there ain't no doctor, so if you're dying, you can't wait, they won't even find your bones, so what are you supposed to do! The clinic here is too difficult. We go to the hospital, and they send us back to the clinic, but why go back there? So, we do it our way. (Helena, 55 years old). We perceive that for the interviewees, "overcoming the barriers", such as accessing medical consultations or medications, and the availability of resources for attendance, and "overcoming obstacles", not only the geographical distances but actually getting to the Center constituted, for the interviewees, great difficulties in accessing the health service. The beliefs that "what's free isn't any good", "only something you pay for has value or can resolve matters", express very well the mistrust and dissatisfaction with the public health service, and motivate the interviewees to seek treatment in the private health network. The feelings of mistrust and dissatisfaction which permeate the interviewees' entire way of thinking lead them to duplication of use of the health services, as found in another study(23), for diagnostic confirmation and checking the therapy. The dissatisfaction with the care model offered by the UESF is owed to the fact that they seek a care centered not only in hard technologies, but also in light technologies ${ }^{(24)}$, for resolving harm caused by becoming ill which is not only functional but, above all, the existential. [...] you need to have plenty of people thinking like this, like you, for things to change (pause) otherwise (shakes head in negation) it carries on the same way, it's like treating cattle, all the same (Wellington, 54 years old, emphasis ours). Humanization has been the guiding principle for re-thinking the health practices, regarding greater comprehensiveness, effectiveness and access $^{(25)}$. The uniquenesses in the way of thinking, associated with the specific characteristics of $\mathrm{SAH}$, mean that the people do not need only physical care, but that they need, above all, encouragement, hope and comprehension.

\section{Conclusion}

In giving relevancy to the social and cultural dimension in the perspective of the person with $\mathrm{SAH}$, we understand that life with the condition is wrapped in a web of meanings, which we seek to interpret through the experience of the condition, which enables us to understand the implicit difficulties in the process of falling ill and in the treatment. In interpreting the experience of the condition for the person with $\mathrm{SAH}$, the following nuclei of meaning appeared: "The condition as expression of the way of living" and "The perspective of cure of the condition". We observed that the nervous problem constitutes the nosological category for the social group researched, a symbolic form of representation which constitutes a harm which is more existential than functional. Different from the findings of other authors - who state that SAH is an invisible condition which does not threaten identity and self-concept - we observed that for the social group researched, it is a visible condition, translated culturally by subjective and objective manifestations, resulting from the urban way of life. We perceived that the causes which are related to the problem reflect the life history more than the histories of the condition. The causes of the nerves, the beliefs and the presence of the problem, which change the person's relationship with the world and with themselves, through the need for adaptation to the new conditions, lead the person to re-signify life with repercussions on the reconstruction of the self-concept and identity. They are supported firmly on the belief of the cure for the problem, however, the recurrences are anticipated, as the external factors 
which trigger the nerves often cannot be avoided, as they are part of life. We observed that the therapeutic routes interpenetrate for the cure of the nervous problem. The family health subsystem is the first treatment resource, in which the manifestations are interpreted, care is given and which serves as guidance and evaluation for the 'folk' and professional health subsystems. The 'folk' health subsystem is an important route due to the cultural identity which is established with the social group researched and because it provides better well-being and remission of symptoms. We observed similarities and divergences between how the people with SAH think and act, and how the health professionals do, whose discourses are defined by cultural meanings determined by the life contexts of both actors who, in the encounter, face unequal dialogs. In adopting the dialogic relationship and valuing the knowledge of common knowledge and the context in which they are integrated, we perceive that powerful cultural beliefs, associated with the notions of the condition and of the treatment which reveal the life experience and the subjectivity in the process of falling ill and of caring for oneself, impede adherence to the pharmacological and non-pharmacological treatment. The gaps evidenced between the point of view of the health professionals and the people with SAH serve us as guidance for re-thinking our praxis, with a view to the reorganization of the work processes in health and the implementation of integrated and contextualized care, which encourages the power of the people with $\mathrm{SAH}$ to live, for empowerment and for self-care.

\section{References}

1. Sociedade Brasileira de Cardiologia. Sociedade Brasileira de Hipertensão. Sociedade Brasileira de Nefrologia. VI Diretrizes Brasileiras de Hipertensão. Arq Bras Cardiol. 2010; 95(Supl1):1-51.

2. Souza SPS de, Lima RAG de. Chronic condition and normality: towards the movement that broadens the power of acting and being happy. Rev. Latino-Am. Enfermagem. jan-fev 2007;15(1):156-64.

3. Prevalência de diabetes e Hipertensão arterial no Brasil. Rev. Saúde Pública 43(Supl2) 74-82. 2009.

4. Ogedegbe G. Labeling and hypertension: it is time to intervene on its negative consequences. Hypertension. 2010;56(3):344-5.

5. Munõz LA, Price $Y$, Gambini L, Stefanelli MC. Significados simbólicos de pacientes com doenças crônicas. Rev Esc Enferm USP. 2003; 37(4):77-84.
6. Tesser CD, Poli Neto P, Campos GWS. Acolhimento e (des)medicalização social: um desafio para as equipes de saúde da família. Ciência Saúde Coletiva. 2010;15(Supl3):3615-24.

7. Langdon EJ, Wiik FB. Anthropology, health and illness: an introduction to the concept of culture applied to the health sciences. Rev. Latino-Am. Enfermagem. mai-jun 2010;18(3):459-66.

8. Franceli $A B$, Figueiredo A, Fava SMCL. Hipertensão arterial: desafios e possibilidades na adesão ao tratamento. REME: Rev Min Enferm. 2008; 12(3):308-12.

9. Fava $S M C L$, Figueiredo AS, Franceli $A B$, Nogueira MS, Cavalari E. Diagnóstico de enfermagem e proposta de intervenções para clientes com hipertensão arterial. Rev Enferm UERJ. 2010;18(4):536-40.

10. Fava SMCLF, Nunes ZB, Gonçalves MFC, Nogueira, MS. Educação em saúde e adesão ao tratamento na perspectiva histórico cultural. Saúde Transform Soc. $2011 ; 2(1): 81-7$.

11. Geertz C. A interpretação das culturas. Rio de Janeiro: Livros Técnicos e Científicos Editora; 1989. 224 p.

12. Kleinman A. The Illness Narratives: Suffering, Healing and the Human Condition. New York: Basic Books; 1988. 269 p.

13. Trad LAB, Tavares JSC, Soares CS, Ripardo RC. Itinerários terapêuticos face à hipertensão arterial em famílias de classe popular. Cad Saúde Pública. 2010;26(4):797-806.

14. Pistulka GM, Winch PJ, Park $H$, Han HR, Kim MT. Maintaining an outward image: a Korean immigrant's life with type 2 Diabetes Mellitus and Hypertension. Qual Health Res.2012;22(6):825-34.

15. Barnes DM, Lu JH. Mexican Immigrants' and Mexican Americans' Perceptions of Hypertension. Qual Health Res. 2012;22(12):1685-93.

16. Aroian KJ, Peters RM, Rudner N, Waser, L. Hypertension Prevention Beliefs of Hispanics. J Transcult Nurs. 2012;23(2):134-42.

17. Pope C, Mays N. Pesquisa Qualitativa na atenção à saúde. Porto Alegre: Artmed; 2009.172 p.

18. Canesqui AM. Olhares socioantropológicos sobre os adoecidos crônicos. São Paulo: HUCITEC; 2007. 149 p.

19. Ferreira $\mathrm{ABH}$. Novo Dicionário Aurélio da Língua Portuguesa. Curitiba: Positivo; 2008.

20. Kleinman A. Patients and healers in the context of culture: an exploration of borderland between Anthropology and Psychiatry. Berkeley: University of California Press; 1980.427 p. 
21. Middleton JL. A proposed new model of hypertensive treatment behavior in African Americans. J Nat Med Assoc. 2009;101(1):12-7.

22. Boff L. Saber cuidar: ética do humano: compaixão pela terra. Petrópolis: Vozes; 1999. $199 \mathrm{p}$.

23. Mishima SM, Paula VGP, Pereira MJBP, Almeida MCP, Kawata LS. (In)Satisfação dos usuários: duplicação e uso simultâneo na utilização de serviços na Saúde da Família. Rev Esc Enferm USP. 2010;44(3):766-73.

24. Merhy EE. Saúde: a cartografia do trabalho vivo. $3^{a}$ ed. São Paulo: Hucitec; 2007.189 p.

25. Marin MJS, Storniolo LV, Moravcik MY. Humanization of Care from the Perspective of the Family Health Strategy Teams in a City in the Interior of São Paulo, Brazil. Rev. Latino-am Enfermagem. jul-ago 2010;18(4):763-9. 